\title{
CORRIGENDUM
}

\section{Identifying polyglutamine protein species in situ that best predict neurodegeneration}

Jason Miller, Montserrat Arrasate, Elizabeth Brooks, Clare Peters Libeu, Justin Legleiter, Danny Hatters, Jessica Curtis, Kenneth Cheung, Preethi Krishnan, Siddhartha Mitra, Kartika Widjaja, Benjamin A Shaby, Gregor P Lotz,

Yvonne Newhouse, Emily J Mitchell, Alex Osmand, Michelle Gray, Vanitha Thulasiramin, Frédéric Saudou, Mark Segal, X William Yang, Eliezer Masliah, Leslie M Thompson, Paul J Muchowski, Karl H Weisgraber \& Steven Finkbeiner

Nat. Chem. Biol. 7, 925-934 (2011); published online 30 October 2011; corrected after print 2 December 2011

In the version of this article initially published, lanes 6 and 8 in Figure $6 \mathrm{~b}$ had identical blot images. The blot now shown in lane 8 has been confirmed to come from the same gel as those in the other lanes and has been used to replace the incorrect blot image in the HTML and PDF versions of the article.

\section{CORRIGENDUM}

\section{Dafadine inhibits DAF-9 to promote dauer formation and longevity of Caenorhabditis elegans}

Genna M Luciani, Lilia Magomedova, Rachel Puckrin, Malene L Urbanus, lain M Wallace, Guri Giaever, Corey Nislow, Carolyn L Cummins \& Peter J Roy

Nat. Chem. Biol. 7, 891-893 (2011); published online 6 November 2011; corrected after print 2 December 2011

In the version of this article initially published, the discovery and synthesis of the dafachronic acids was not attributed properly in two instances. On p. 892, the statement "the DAF-9 cytochrome P450 completes the synthesis of the dafachronic-acid hormones ${ }^{11,13 \text { " }}$ should have cited references 11, 13 and 14, and the statement "DAF-9 catalyzes two sequential oxidation steps at the C26 position to generate metabolites that are first hydroxylated and then carboxylated to products named $\Delta^{4}$ - and $\Delta^{7}$-dafachronic acid, respectively ${ }^{13}$ " should have cited reference 14 instead of 13. The errors have been corrected in the PDF and HTML versions of this article.

\section{CORRIGENDUM}

\section{Click-generated triazole ureas as ultrapotent in vivo-active serine hydrolase inhibitors}

Alexander Adibekian, Brent R Martin, Chu Wang, Ku-Lung Hsu, Daniel A Bachovchin, Sherry Niessen, Heather Hoover \& Benjamin F Cravatt

Nat. Chem. Biol. 7, 469-478 (2011); published online 15 May 2011; corrected after print 15 February 2012

In the version of this article initially published, the authors concluded, on the basis of the substantial (approximately five-fold) N1 regioselectivity observed for reactions that form the unsubstituted triazole ureas shown in Figures 1-3, that the major regioisomeric product for the 4-substituted triazole ureas shown in Figures 1 and 3 was also the N1 regioisomer. They have since determined by X-ray crystallography (provided as Supplementary Data Sets 1 and 2) that the N2 regioisomer is the major product for the 4-substituted triazole ureas. The structures have been corrected in the HTML and PDF versions of the article and in the chemical probe table associated with the article. 